Relations industrielles

Industrial Relations

\title{
Cohen-Rosenthal, Edward, and Cynthia E. Burton, Mutual Gains. A Guide to Union-Management Cooperation
}

\section{Colette Bernier}

Volume 49, numéro 2, 1994

URI : https://id.erudit.org/iderudit/050945ar

DOI : https://doi.org/10.7202/050945ar

Aller au sommaire du numéro

Éditeur(s)

Département des relations industrielles de l'Université Laval

ISSN

0034-379X (imprimé)

1703-8138 (numérique)

Découvrir la revue

Citer ce compte rendu

Bernier, C. (1994). Compte rendu de [Cohen-Rosenthal, Edward, and Cynthia E. Burton, Mutual Gains. A Guide to Union-Management Cooperation]. Relations industrielles / Industrial Relations, 49(2), 406-408.

https://doi.org/10.7202/050945ar

Tous droits réservés (C) Département des relations industrielles de l'Université Laval, 1994
Ce document est protégé par la loi sur le droit d'auteur. L'utilisation des services d'Érudit (y compris la reproduction) est assujettie à sa politique d'utilisation que vous pouvez consulter en ligne.

https://apropos.erudit.org/fr/usagers/politique-dutilisation/ 
predictable. Notwithstanding this minor shortcoming, this is a very good book and I highly recommend it.

Joseph B. Rose

McMaster University

Mutual Gains, A Guide to Union-Management Cooperation, by Edward CoHeNRosentHal, and Cynthia E. Burton, Ithaca, New York, ILR Press, 2nd ed. rev. 1993, 344 p., ISBN 0-87546-312-6.

Au moment où les expériences de coopération patronale-syndicale deviennent un sujet d'actualité, cette seconde édition mise à jour du livre « Mutual Gains » devrait connaître quelque succès. Ensemble, les auteurs ont travaillé à titre de consultants depuis plus de dix ans auprès d'organismes avec lesquels ils ont implanté divers projets de coopération patronale-syndicale. L'ouvrage ne se veut pas une analyse de la coopération mais bien un guide à l'intention de praticiens; il veut dire pourquoi et surtout comment coopérer pour en faire bénéficier les deux parties. D'ailleurs, les auteurs affirment dès l'entrée de jeu leur penchant tant pro-syndical que pro-patronal. L'expérience de travail d'un des auteurs dans certains organismes syndicaux ressort passablement du livre et en fait, à notre avis, un document aussi intéressant pour les syndicats que pour les gestionnaires et les consultants d'entreprises.

Au premier chapitre, les auteurs tentent de définir la coopération comme un des aspects des relations industrielles, parallèlement à son aspect conflictuel. Ils s'opposent ainsi à une certaine vision qui décrirait la coopération comme un changement radical de la culture managériale ouvrant la voie à une ère de paix sociale en Amérique du Nord. Bien que rapide, leur historique de la coopération patronale-syndicale aux États-Unis a au moins le mérite de montrer que ce phénomène a toujours pris une importance grandissante durant les périodes de crise économique et sociale, pour souvent disparaître par la suite.

Les auteurs définissent la coopération comme étant tout d'abord un objectif commun que syndicats et patronat peuvent identifier et réaliser conjointement. Mais, ajoutent-ils, la coopération ne veut pas dire la perte d'identité de chacune des parties, pas plus que l'absence de conflits. En cela, l'approche du livre se distancie de certaines tentatives patronales qui, de l'avis même des auteurs, voudraient se servir de la voie de la coopération pour mettre de côté les syndicats. Au contraire, et c'est l'idée maitresse du second chapitre, la relation de négociation telle qu'articulée dans la convention collective devrait constituer le fondement de tout projet de coopération. Et le ton du livre devient vite personnalisé lorsque les auteurs tentent de montrer, à l'intention aussi bien des syndicats (chapitre 2) que des directions d'entreprises (chapitre 3), les risques et les bénéfices de la coopération.

Ensuite, la seconde partie du livre tente de dresser un tableau des divers types de coopération patronale-syndicale, à différents niveaux. Ainsi, fidèles à leur approche voulant que la négociation collective soit le premier mécanisme de coopération syndicale-patronale en Amérique du Nord, les auteurs s'intéressent d'abord aux 
nouveaux mécanismes de négociation et de résolution de problèmes, tels par exemple les processus de médiation et de conciliation. Le chapitre suivant discute plus spécifiquement des différents comités patronaux-syndicaux mis en place dans les entreprises. On y traite ainsi dans l'ordre, des comités de santé et de sécurité au travail, de loin les plus nombreux en Amérique du Nord, des comités de formation professionnelle, des comités d'assistance et de bien-être, des comités sur l'environnement et l'énergie, des comités sur les changements technologiques ainsi que des comités d'analyse de la valeur. Les auteurs abordent chacun de ces types de comités en expliquant leur origine et leur évolution dans différents pays sur la base de nombreux exemples (notons en passant que certains exemples canadiens - ontariens - sont commentés).

Aux termes de cette description, le lecteur reste un peu sur sa faim : on nous a présenté l'un après l'autre différents types de comités mais sans les classer d'une quelconque façon. Une démarche plus analytique aurait pu rendre les descriptions de ce chapitre plus intéressantes. Par exemple, on aurait eu avantage à cibler les restructurations actuelles et l'importance relative qu'ont pris certains comités dans la période récente (pensons aux comités sur les changements technologiques ou encore aux comités sur la formation professionnelle).

Il faudra attendre au chapitre suivant, le chapitre 6, qui porte sur le développement de nouvelles structures d'organisation (des cercles de qualité au redesign sociotechnique) pour entrer enfin dans l'actualité du sujet. Il y est question des expériences directes de participation sur les lieux de travail à partir des restructurations récentes du travail. Nous découvrons ici le plan implicite de l'ouvrage (qui aurait gagné à être explicité) et qui, après avoir abordé la coopération aux niveaux formels de la négociation et des comités structurés, l'aborde maintenant au niveau de la participation directe et souvent moins formelle sur les lieux mêmes de travail.

Ce chapitre est intéressant à plus d'un titre. D'abord, il fournit un assez bon panorama de l'évolution des expériences de restructuration impliquant une participation directe des travailleurs. Les auteurs l'abordent en tant que praticiens et l'on sent une grande expérience de terrain derrière les nombreux exemples relatés, qu'ils soient pris dans la littérature ou dans leur propre bagage de praticiens. Chacune des formes abordées (des cercles de qualité au juste-à-temps, en passant par les équipes de travail semiautonomes) est bien définie et souvent replacée dans le contexte de son évolution historique. Les avantages et désavantages de chacune de ces formes sont bien documentés et, ce qui est rare, les auteurs donnent une place importante et bien articulée à la vision syndicale.

Dans sa première édition, cette seconde partie du livre se terminait par un chapitre sur les expériences de coopération concernant le marketing, le service à la clientèle et le développement de produits en partant de l'idée que les employés sont souvent plus au fait des besoins des clients que la haute direction des entreprises. Dans la dernière édition, les auteurs ont ajouté un chapitre sur les comités conjoints de qualité. Après avoir discuté de l'évolution du concept, de ses avantages et désavantages, les auteurs avancent l'idée d'appliquer les principes de qualité à la convention collective pour éviter que les professionnels des relations du travail soient exclus des processus en cours en continuant à travailler avec un modèle conflictuel de relations du travail. 
La troisième partie du livre porte sur le « comment faire ". Elle aborde les questions de la préparation à la mise en place d'un comité, de son fonctionnement (le planifier, entraîner les parties à le gérer, etc.) et de son évaluation. Les auteurs se défendent d'avoir écrit un livre de recettes puisque chaque application peut varier par rapport à une autre; il s'agit plutôt de soulever les questions à se poser face à chaque nouvel essai. Dans cette partie du livre, les conseils donnés proviennent encore d'une solide expérience des auteurs comme consultants.

La dernière partie du livre s'intéresse aux systèmes alternatifs de rémunération visant à « partager le gâteau ». L'implication des salariés dans les nouvelles expériences de travail contribue souvent à reconsidérer les modèles traditionnels de rémunération. Sont ainsi présentées différentes formes plus ou moins nouvelles de rémunération tels le salaire aux pièces, individuel ou collectif, ou encore la rémunération basée sur les connaissances. On y présente ensuite certains plans américains de partage des profits et finalement certaines expériences de propriété d'entreprises par les travailleurs. Pour chaque modèle, une définition est apportée alors que les auteurs tentent d'en faire ressortir les aspects positifs et négatifs. Selon eux, une bonne paie ne suffit pas à créer un bon milieu de travail, pas plus que la participation seule n'est suffisante. Liés ensemble, ces deux éléments pourraient susciter, toujours selon les auteurs, une bonne « qualité de vie au travail ».

Cette référence assez constante dans le livre à la qualité de vie au travail, bien que les auteurs en parlent comme d'un concept générique, pourrait agacer certains syndicats, quand on sait le peu de succès que les programmes du même nom ont eu. Par ailleurs, comme on l'a dit souvent, les auteurs ont su faire une bonne place à la vision syndicale, surtout quand ils abordent les différents pièges des expériences présentées. Aussi, peut-on conclure qu'il s'agit d'un bon guide qui pourrait être utile autant à la partie syndicale qu'à la partie patronale dans les entreprises qui veulent mettre sur pied des programmes conjoints. Le livre présente de fait un très bon survol des différents concepts, il sait les illustrer à l'aide d'exemples et en faire ressortir les « pour » comme les « contre ». En ce sens, il peut aussi être utile à des étudiants ou étudiantes qui voudraient se familiariser avec ces notions et ces pratiques. Par contre, celui ou celle qui y chercherait une analyse poussée du phénomène de la participation patronale-syndicale ou des nouvelles formes d'organisation du travail serait déçu. D'ailleurs, la plus grande critique que l'on pourrait adresser à l'ouvrage serait sans doute ses lacunes au niveau de l'analyse. Mais s'il ne s'agit pas d'un livre de réflexion sur ces expériences, ça n'était pas là non plus l'intention première des auteurs.

Colette BERnier

Université Laval

Les relations du travail au Québec : une analyse de la situation dans le secteur public, par Michel Leclerc et Michel QuIMPER, Sainte-Foy, Presses de 1'Université du Québec, 1994, 336 p., ISBN 2-7605-0723-8.

Les auteurs présentent cet ouvrage comme « un survol rapide des relations du travail, en guise d'initiation générale et d'invitation à aller plus loin " (p. XIX). 